\title{
Luminescence in Mn-doped CdS nanocrystals
}

\author{
ANGSHUMAN NAG ${ }^{1}$, SAMEER SAPRA ${ }^{1}$, SUBHRA SEN GUPTA ${ }^{1}$, ANKITA PRAKASH ${ }^{1}$, \\ AJIT GHANGREKAR ${ }^{2}$, N PERIASAMY ${ }^{2}$ and D D SARMA ${ }^{1,3, *}$ \\ ${ }^{1}$ Solid State and Structural Chemistry Unit, Indian Institute of Science, Bangalore 560 012, India \\ ${ }^{2}$ Department of Chemical Sciences, Tata Institute of Fundamental Research, Mumbai 400 005, India \\ ${ }^{3}$ Centre for Advanced Materials, Indian Association for the Cultivation of Science, Kolkata 700 032, India
}

\begin{abstract}
We have synthesized Mn-doped CdS nanocrystals (NCs) with size ranging from 1.8-3 nm. Photoluminescence (PL) spectra of the doped NCs differ from that of the undoped NCs with an additional peak due to $\mathrm{Mn} d-d$ transitions. Electron paramagnetic resonance spectra along with $\mathrm{X}$-ray absorption spectroscopy and PL spectra confirm the incorporation of Mn in the CdS lattice. The fact that emissions from surface states and the Mn $d$ levels occur at two different energies, allowed us to study the PL lifetime decay behaviour of both kinds of emissions.
\end{abstract}

Keywords. Luminescence; doping; semiconductor nanocrystals.

\section{Introduction}

Doped semiconductor nanocrystals are being studied intensively to achieve two different material properties, viz. luminescence and magnetism. The impurity atoms doped sparsely into nanocrystals provide sharp atomic levels for capturing the excited electrons and holes, formed upon excitation of particles across the band gap, from the host semiconductor lattice and displaying narrow emission spectra due to recombination of electrons and holes between these sharp levels. If the impurity atoms have unfilled $d$ electrons, then one also observes the magnetic properties. Either or both of these properties have been studied in a few Mn-doped semiconductors such as $\mathrm{Cd}_{1-x} \mathrm{Mn}_{x} \mathrm{~S}$ (Levy et al 1996; Counio et al 1998; Feltin et al 1999; Nag et al 2007), $\mathrm{Zn}_{1-x} \mathrm{Mn}_{x} \mathrm{~S}$ (Wang et al 1991; Bhargava et al 1994; Soo et al 1994; Sapra et al 2003), $\mathrm{Cd}_{1-x} \mathrm{Mn}_{x} \mathrm{Se}$ (Erwin et al 2005), $\mathrm{Zn}_{1-x} \mathrm{Mn}_{x} \mathrm{Se}$ (Pradhan and Peng 2007), $\mathrm{Zn}_{1-x} \mathrm{Mn}_{x} \mathrm{O}$ (Viswanatha et al 2004), $\mathrm{Ga}_{1-x} \mathrm{Mn}_{x} \mathrm{As}$ and $\mathrm{In}_{1-x} \mathrm{Mn}_{x} \mathrm{As}$ (Ohno 1998, 1999).

Manganese-doped CdS nanocrystals have been mainly studied due to their luminescence properties. This is due to the fact that the doped $\mathrm{Mn}^{2+}$ ions provide good traps for the excited electrons though the Mn spins do not align to give magnetic behaviour in the case of bulk $\mathrm{Cd}_{1-x} \mathrm{Mn}_{x} \mathrm{~S}$; it gives rise to paramagnetism (Furdyna 1988). However, in the nanocrystalline phase, Hoffman and coworkers (2000) have reported the presence of a giant internal magnetic field, with a commensurate Zeeman splitting of the electron and hole levels as high as $100 \mathrm{meV} . \mathrm{Mn}^{2+}$ ion emission has been reported at $\sim 590 \mathrm{~nm}$ (Wang et al 1991;

*Author for correspondence (sarma@sscu.iisc.ernet.in)
Bhargava et al 1994; Soo et al 1994; Levy et al 1996; Counio et al 1998; Feltin et al 1999; Sapra et al 2003; Erwin et al 2005; Nag et al 2007; Pradhan and Peng 2007). The excited electrons are transferred from the conduction band to the $\mathrm{Mn} d$ levels through which the radiative de-excitation occurs via ${ }^{4} T_{1}-{ }^{6} A_{1}$ transitions. This is observed as the orange emission. Earlier reports show a broad band apart from the orange band in the doped and undoped $\mathrm{CdS}$ nanocrystals at a wavelength higher than $600 \mathrm{~nm}$. This has been attributed to defect or surface related emission. In the present report, we observe this emission at wavelength lower than the Mn emission. We attribute this emission to the defect states and the observation of this emission at a higher energy to the difference in the method of synthesis and is described below in the discussion on fluorescence studies.

\section{Experimental and methods}

The chemicals were used as received without further purification. $\mathrm{Cd}(\mathrm{OAc})_{2} \cdot 2 \mathrm{H}_{2} \mathrm{O}, \mathrm{Mn}(\mathrm{OAc})_{2} \cdot 4 \mathrm{H}_{2} \mathrm{O}$ and solvent dimethyl sulphoxide (DMSO) were purchased from Ranbaxy. $\mathrm{Na}_{2} \mathrm{~S} \cdot 9 \mathrm{H}_{2} \mathrm{O}$ was obtained from Aldrich and the capping agent, 1-thioglycerol, from Fluka. Double distilled water was employed for all the reactions. Synthesis procedure was borrowed from Nag et al (2007) but with a desired change in the reaction temperatures. A typical synthesis involved dissolution of $\mathrm{Cd}^{2+}(4-y \mathrm{mmol})$ and $\mathrm{Mn}^{2+}(y \mathrm{mmol})$ salts in $40 \mathrm{ml}$ DMSO and $0.5 \mathrm{ml}$ thioglycerol was added to this solution. The quantity, $y$, was equated to the Mn percentage, $x$ as in $\mathrm{Cd}_{1-x} \mathrm{Mn}_{x} \mathrm{~S}$, using the formula $x=(y / 4)$. A separate solution of $\mathrm{S}^{2-}$ in $10 \mathrm{ml}$ water was freshly prepared and added drop by drop to the DMSO solution over a period of $\sim 5 \mathrm{~min}$. Then the reaction 
mixture was heated to the desired temperature for $12 \mathrm{~h}$. Acetone was added to the reaction mixture to precipitate the nanocrystals which were then washed with methanol several times to remove the adhering unreacted reagents. Dilute solutions in DMSO-water mixture were prepared for UV-Vis and fluorescence studies.

UV-Vis absorption experiments were performed on a Perkin Elmer's Lambda 35 UV/visible spectrometer at a bandwidth of $2 \mathrm{~nm}$. Fluorescence emission was recorded on a Perkin Elmer LS 55 with the excitation and emission slits at $5 \mathrm{~nm}$ each. Excitation was carried out at $370 \mathrm{~nm}$. Powder X-ray diffraction (XRD) patterns were recorded on a Siemens D5005 X-ray diffractometer at $1.2^{\circ}$ per min. Electron paramagnetic resonance (EPR) spectra were measured on powder samples in a quartz tube. X-ray absorption spectra (XAS) were recorded at the BEAR beamline at Sincrotrone centre, Elettra, Trieste, Italy.

The theoretical $2 p \rightarrow 3 d$ XAS of Mn in Mn-doped CdS was calculated using the Lanczos iterative algorithm for a many-body Hamiltonian, based on a fully coherent spectral function for the $\left(\mathrm{MnS}_{4}\right)^{6-}$ tetrahedral cluster corresponding to the zinc-blende or the wurtzite structure. The details of the Hamiltonian involved and the method of calculation are discussed elsewhere (Mahadevan and Sarma 2000). The calculations were performed with complete Mn $3 d-3 d$ and $2 p-3 d$ multiplet interactions, corresponding to average values $\left(U_{d d}\right.$ and $\left.U_{p d}\right)$ of $5.8 \mathrm{eV}$ and $7 \mathrm{eV}$, respectively (Bocquet et al 1992). The hopping parameters used for MnS were $\mathrm{S}-\mathrm{S}(p p \sigma)=0.32 \mathrm{eV}, \mathrm{S}-\mathrm{S}(p p \pi)=$ $-0.04 \mathrm{eV}, \mathrm{S}-\mathrm{Mn}(p d \sigma)=-1.64 \mathrm{eV}$, and S-Mn $(p d \pi)=$ $0.42 \mathrm{eV}$. The charge transfer energy $(\Delta=5 \mathrm{eV})$ and the crystal field $\left(T_{\mathrm{d}}\right)$ strength $(10 \mathrm{Dq}=0.4 \mathrm{eV})$, were adjusted to obtain the best description of the experimental data. The spectrum thus obtained was broadened by a Gaussian function of width, $0.5 \mathrm{eV}$, to simulate instrumental broadening and a Lorentzian function of width, $0.55 \mathrm{eV}$, to account for the finite lifetime of the core hole.

\section{Results and discussion}

Figure 1 shows the low and wide-angle X-ray diffraction (XRD) patterns of nanocrystals of CdS synthesized at $60^{\circ} \mathrm{C}, 100^{\circ} \mathrm{C}, 130^{\circ} \mathrm{C}$ and $160^{\circ} \mathrm{C}$. Also shown are the XRD patterns of bulk CdS in the wurtzite and zinc-blende structure. Upon decreasing the reaction temperature, one observes that the XRD peaks get broader. This indicates that the size of the nanocrystals is small when grown at lower temperatures, whereas higher temperatures yield larger sized particles. The diameter, $D$, of the nanocrystals can be determined using the Scherrer formula (Guinier 1963)

$$
D=\frac{4}{3} \frac{0 \cdot 9 \lambda}{B \cos \theta}
$$

where $B$ is the full-width at half maximum (FWHM) of the XRD peak at $2 \theta$, recorded with an incident wavelength, $\lambda$. The bulk XRD pattern is broadened to an amount determined by the variable parameter, $B$, to get the best description of the nanocrystal XRD pattern. This tells us about the size of the nanocrystals. These sizes are tabulated in table 1. The bulk patterns that are broadened and compared with the experimental results are shown in the bottom of the figure. No single broadened bulk pattern can match any of the experimental patterns. Hence, we

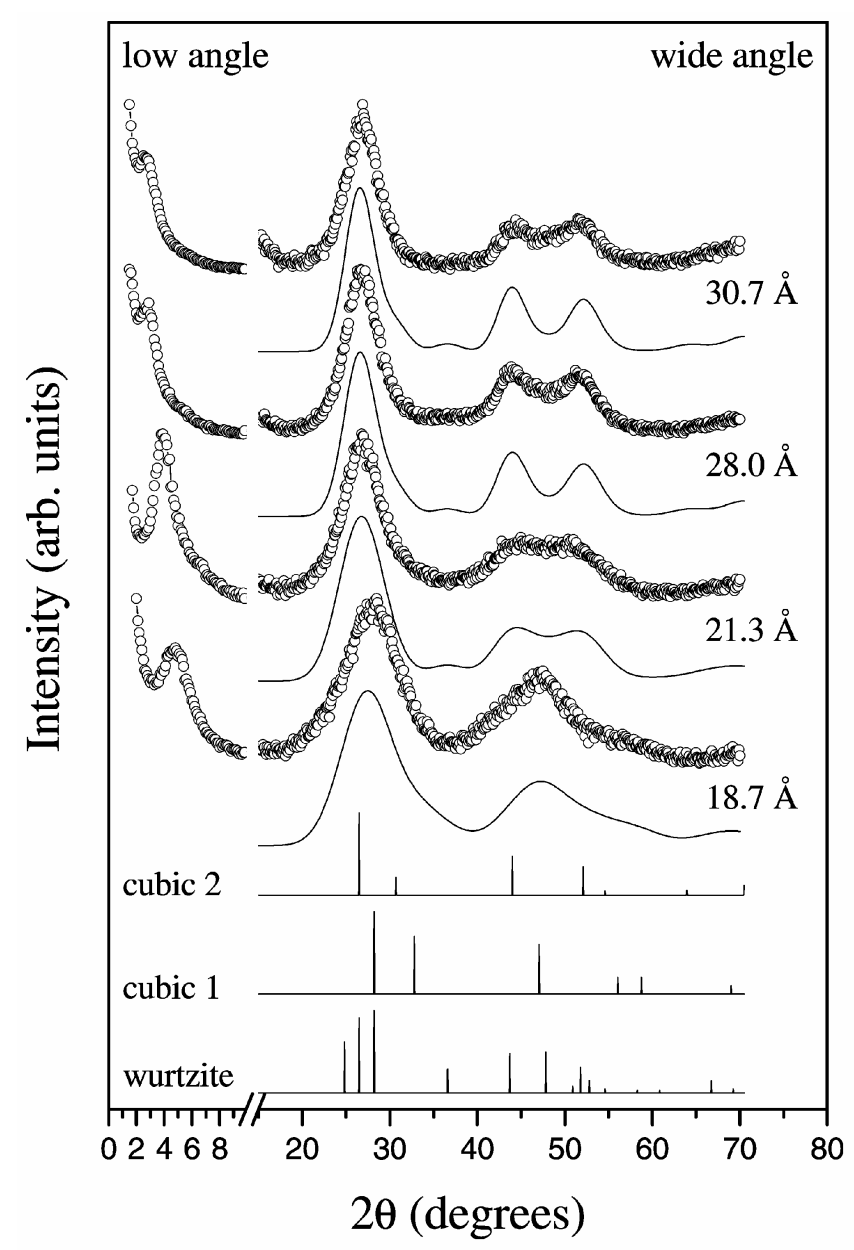

Figure 1. Low angle and wide angle $\mathrm{X}$-ray diffraction patterns for undoped $\mathrm{CdS}$ nanocrystals synthesized at $60^{\circ} \mathrm{C}, 100^{\circ} \mathrm{C}$, $130^{\circ} \mathrm{C}$ and $160^{\circ} \mathrm{C}$. The bulk patterns for cubic (cubic 1 and cubic 2) and wurtzite, that are used for broadening, are shown at the bottom of the figure. Below each wide angle XRD, the corresponding broadened pattern is also shown.

Table 1. Sizes obtained from different characterization techniques. All values are in Ångströms.

\begin{tabular}{llll}
\hline $\begin{array}{l}\text { Temperature } \\
\left({ }^{\circ}\right) \mathrm{C}\end{array}$ & $\begin{array}{c}\text { Wide angle } \\
\text { XRD }\end{array}$ & $\begin{array}{c}\text { Low angle } \\
\text { XRD }\end{array}$ & UV-Vis \\
\hline 60 & $18 \cdot 7$ & $18 \cdot 4$ & $19 \cdot 5$ \\
100 & $21 \cdot 3$ & $22 \cdot 6$ & $22 \cdot 5$ \\
130 & $28 \cdot 0$ & $30 \cdot 4$ & $27 \cdot 7$ \\
160 & $30 \cdot 7$ & $35 \cdot 3$ & $30 \cdot 0$ \\
\hline
\end{tabular}


used a superposition of the broadened bulk patterns in varying proportions to obtain an agreement with the experimental data. It is found that the samples made at higher temperatures, $100^{\circ} \mathrm{C}, 130^{\circ} \mathrm{C}$ and $160^{\circ} \mathrm{C}$, are a mixture of the cubic zinc blende (cubic 2) and the hexagonal wurtzite structure. The samples prepared at $60^{\circ} \mathrm{C}$ crystallize in the zinc-blende structure, though they are a mixture of two cubic structures viz. cubic 1 (space group: $F m 3 m$ ) and cubic 2 (space group: $F \overline{4} 3 m$ ) as shown in the figure. It has been shown earlier that phase transformations occur at very small nanocrystal sizes (Vossmeyer et al 1994).

The nanocrystals tend to organize themselves into ordered structures. This gives rise to diffraction peaks in the very low angle regime, corresponding to the average distance between the nanocrystals. The left part of figure 1 shows the low angle diffraction peaks from the four different samples. The distance between two nanocrystal centres corresponding to the low angle peak is given by the Bragg's Law. From the low angle peaks for the $\mathrm{CdS}$, the sizes calculated for the samples synthesized at various temperatures are listed in table 1 . The values are in good agreement with those obtained from wide angle XRD.

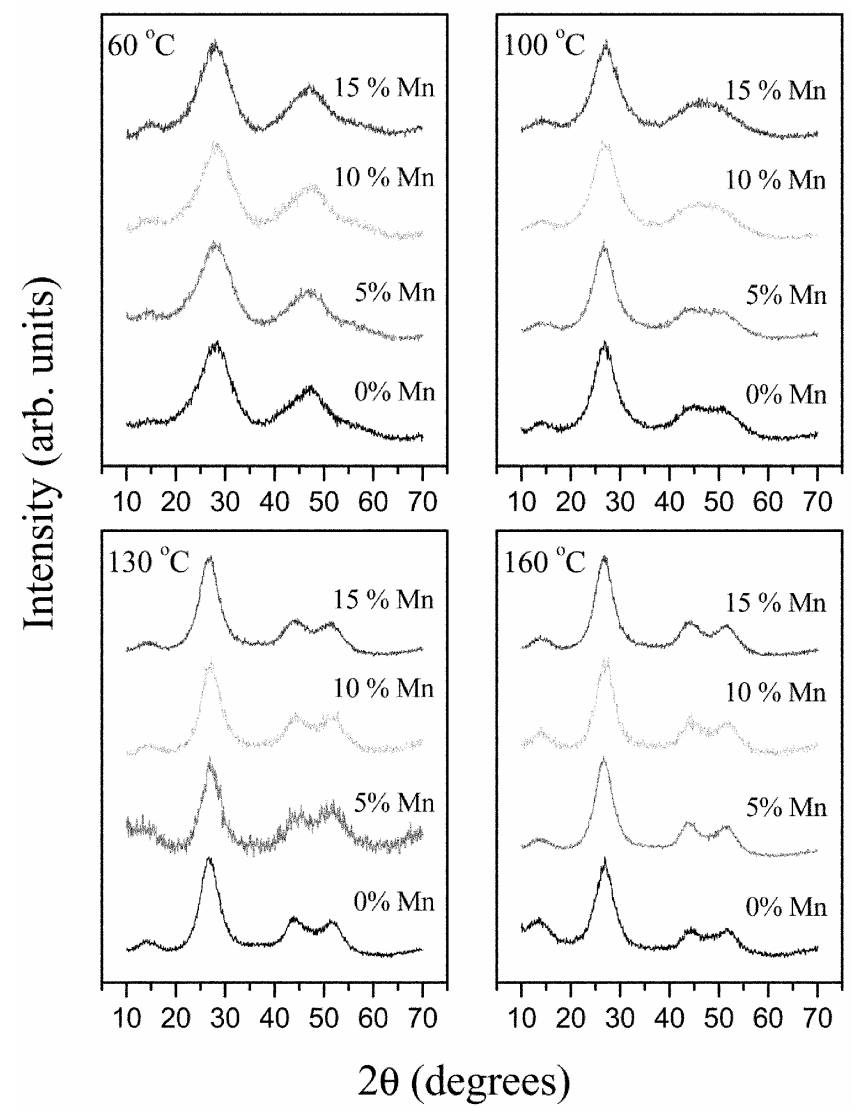

Figure 2. Wide angle X-ray diffraction patterns for varying amounts of $\mathrm{Mn}$ in Mn-doped CdS nanocrystals synthesized at different temperatures. The broad peak around 14 degree is due to the amorphous glass substrate used as a sample holder.
The XRD patterns do not change upon doping $\mathrm{Mn}^{2+}$ ions into the CdS nanocrystals. This is shown by the wide angle $\mathrm{X}$-ray diffraction patterns for $\mathrm{Cd}_{1-x} \mathrm{Mn}_{x} \mathrm{~S}$ nanocrystals in figure 2 .

Figure 3 shows the UV-Vis spectra of the four CdS samples synthesized at different temperatures. We notice a systematic shift in the absorption edge, away from bulk $\mathrm{CdS}$, as the synthesis temperature is lowered. As already seen from the XRD analysis, the size of the nanocrystals decreases as the reaction temperature is decreased. Thus, due to the quantum confinement effect, the bandgap of the nanocrystals, which is determined from the UV-Vis spectra, increases with a decrease in the particle size. The nanocrystal size can be determined from the bandgap shift seen in the absorption spectra by comparing it with theoretical models. The most accurate model for determining the electronic structure of II-VI nanocrystals uses the tight-binding approximation with the $s p^{3} d^{5}$ orbital basis and next nearest neighbour interactions between the anions and the cations (Sapra et al 2002; Sapra and Sarma 2004). The sizes obtained from the bandgap variation versus size curve for CdS in Sapra and Sarma (2004) are listed in table 1 . The values are in good agreement

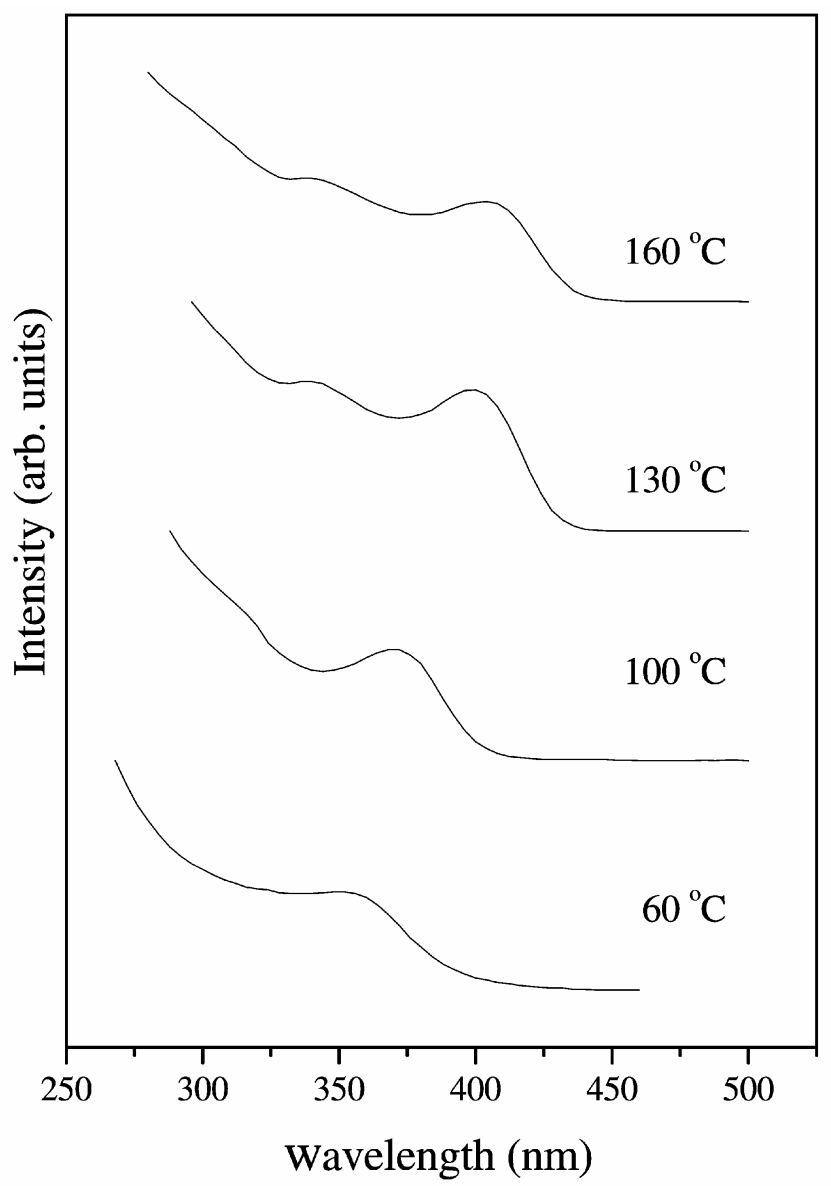

Figure 3. UV-Vis absorption spectra for undoped CdS nanocrystals synthesized at various temperatures. 
with those obtained from the wide and low angle XRD patterns, similar to the ones reported earlier (Nag et al 2007). However, we notice that the size estimate from the low angle XRD patterns is slightly larger compared to the size estimate from the UV-Vis absorption spectra except for the smallest sized clusters. We attribute it to the fact that the lower temperature used $\left(60^{\circ} \mathrm{C}\right)$ for synthesizing the small sized sample gives a rather large size distribution and hence the UV-Vis spectrum has a tail towards the higher wavelength owing to the presence of larger nanocrystals obtained in this synthesis technique, thus giving a slightly larger size estimate. Analogous to $\mathrm{Nag}$ et al (2007), the UV-Vis absorption spectra for identically sized $\mathrm{Mn}$-doped CdS nanocrystals with various Mn percentages (not shown here) indicate that Mn-doping does not change the size of the nanocrystals thus, enabling us to study the properties of fixed size nanocrystals with varying doping concentrations.

Mn-doping into semiconductor nanocrystals has been a topic of debate for the past few years (Wang et al 1991; Bhargava et al 1994; Soo et al 1994; Levy et al 1996; Counio et al 1998; Feltin et al 1999; Sapra et al 2003; Erwin et al 2005; Nag et al 2007; Pradhan and Peng 2007).

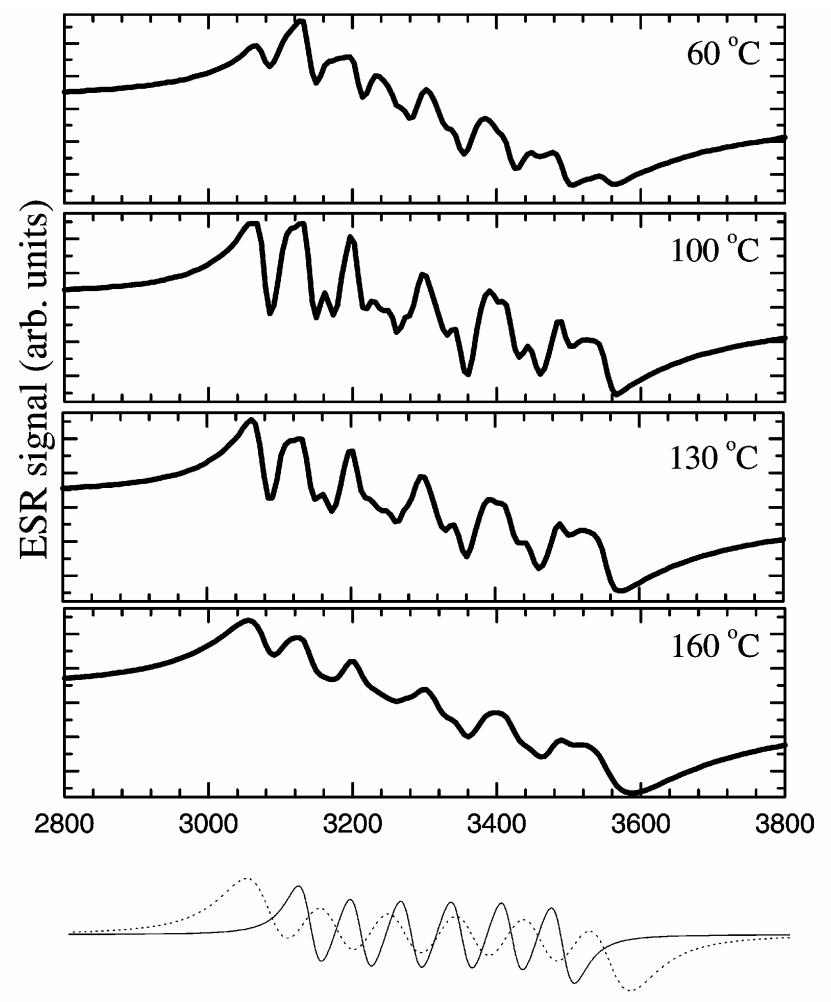

Magnetic Field (Gauss)

Figure 4. Electron paramagnetic resonance spectra for $\mathrm{Mn}$ doped CdS nanocrystals synthesized at different temperatures. The nominal amount of $\mathrm{Mn}$ is $10 \%$. At the bottom of the figure the two components with splitting of 69 and 90 Gauss are shown.
Techniques such as X-ray diffraction, electron paramagnetic resonance, EXAFS and fluorescence emission have been employed to establish the presence of Mn centres in the nanocrystal lattice. Although lattice dilation upon incorporation of impurity atoms is conclusive to establish the presence of $\mathrm{Mn}$ inside the nanocrystals, the other methods are not as conclusive, though they are indicative. In case of very small sized nanocrystals, where the XRD peaks are broad, and in addition, the amount of dopant is very small, it is impossible to determine the shifts in the XRD pattern. However, a careful preparation of the sample and detection of the presence of Mn using various techniques can establish the Mn centres being present in the host CdS nanocrystal.

The presence of Mn ions in the nanocrystals is evidenced from the EPR spectra shown in figure 4 . The figure shows the EPR spectra of different sized CdS nanocrystals doped with varying amounts of Mn. The spectra suggest a multiple finger pattern characteristic of the $\mathrm{Mn}^{2+}$ species. Thus, one can confidently state that $\mathrm{Mn}^{2+}$ ions are present in the nanocrystals. Moreover, these $\mathrm{Mn}^{2+}$ ions are spatially isolated because we observe the sextet patterns; clustered $\mathrm{Mn}^{2+}$ ions give rise to broad and featureless EPR spectra due to Mn-Mn interactions (Sapra et al 2003). A closer inspection of the EPR spectra shows that the spectra itself is composed of atleast two different types of signals. This can be attributed to the fact that $\mathrm{Mn}^{2+}$ ions are present in different environments (Nag et al 2007). Inside the nanocrystals the $\mathrm{Mn}^{2+}$ ions can substitute for the $\mathrm{Cd}^{2+}$ ions which are tetrahedrally coordinated. If $\mathrm{Mn}^{2+}$ ions are present on the surface of the nanocrystals, then they would prefer the octahedral coordination. The tetrahedral coordination gives rise to an EPR sextet with a peak-to-peak separation of 69 Gauss and the octahedral environment leads to an approximately 90 Gauss separation. Both these components are present in the EPR spectra recorded from the $\mathrm{Cd}_{1-x} \mathrm{Mn}_{x} \mathrm{~S}$ samples, similar to those reported earlier (Nag et al 2007). The individual components are also shown in the bottom part of the figure. We notice that the samples synthesized at $60^{\circ} \mathrm{C}$ have the maximum amount of the 69 Gauss component which decreases as we go towards samples synthesized at higher temperatures. We believe that higher synthesis temperatures drive the $\mathrm{Mn}^{2+}$ ions towards the surface of the nanocrystals.

We have employed yet another method to establish the presence of $\mathrm{Mn}^{2+}$ ions in CdS nanocrystals. X-ray absorption spectroscopy (XAS) is a useful technique to study the local environment (coordination) and oxidation state of various ions. In particular, the transition metal ions have been well characterized (van der Laan and Kirkman 1992). Figure 5 shows the XAS for the Mn $L_{3}$ edge for the highest doped samples as specified in the figure. The spectral shape resembles that of $\mathrm{Mn}^{2+}$ ions in the interior of $\mathrm{ZnO}$ nanocrystals (Mikulec et al 2000). In order to determine the valency, symmetry, and the crystal field strength of 
the $\mathrm{Mn}^{2+}$ ions in the present case, we carried out theoretical calculations for various crystal field strengths. The spectrum that gives the best description of the experimental spectrum corresponds to a $\mathrm{Mn}^{2+}$ species in a tetrahedral symmetry $(10 \mathrm{Dq}=0.4 \mathrm{eV})$ and is shown in the figure with a solid line, evidently providing a good agreement with the experimental data. However, we must point out that even the octahedral symmetry with the same crystal field strength provides an equally good description to the experimental spectrum (van der Laan and Kirkman 1992). Thus, $\mathrm{Mn}^{2+}$ ions can be inside or at the surface of the nanocrystals and could not distinguish between the two kinds of $\mathrm{Mn}^{2+}$ species. It is interesting to note that the characteristic Mn $2 p$ spectrum for a tetrahedrally coordinated $\mathrm{Mn}^{2+}$ ion is observed only in the case of the highest doped sample. Thus, even $20 \%$ nominal $\mathrm{Mn}^{2+}$ doping gives a very low concentration of $\mathrm{Mn}^{2+}$ ions in the nanocrystals.

Figure 6 shows the fluorescence emission spectra of undoped and Mn-doped CdS nanocrystals with different amounts of $\mathrm{Mn}$ concentration, synthesized at different temperatures. The excitation wavelengths used are at the absorption band edges of the respective samples. Un-

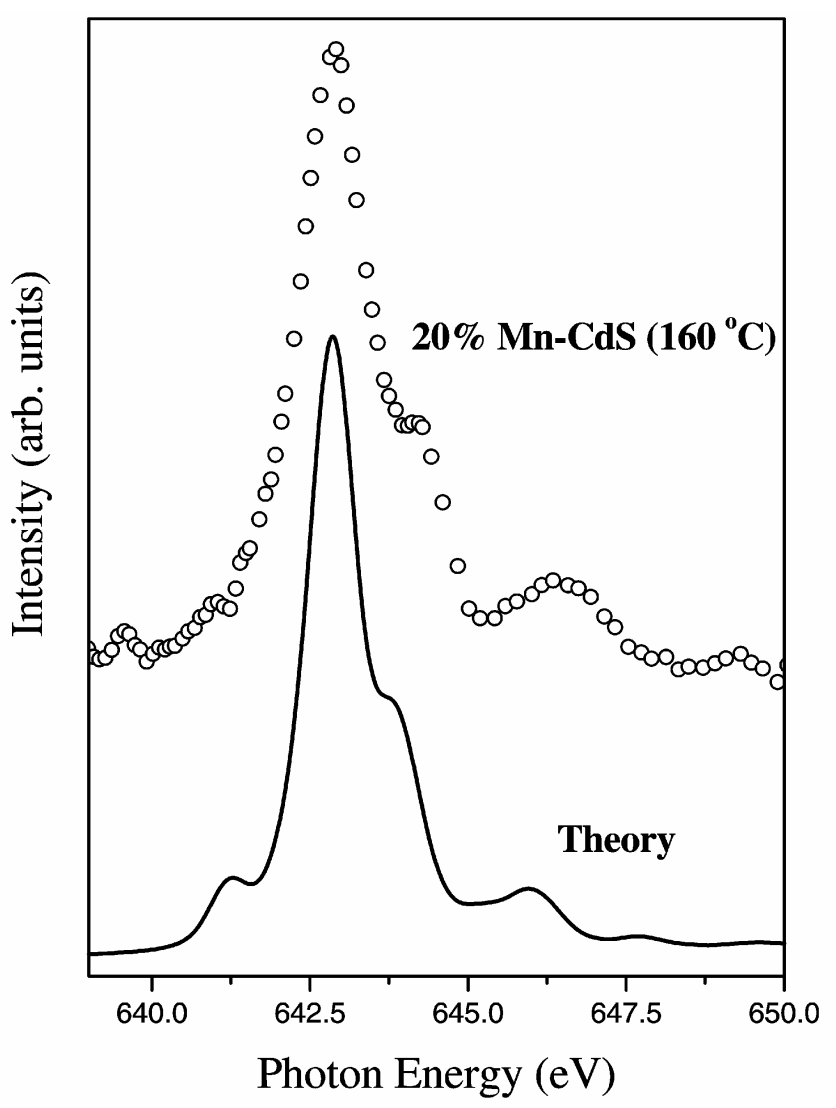

Figure 5. X-ray absorption for the $\mathrm{Mn} L_{3}$ edge for the highest concentration of $\mathrm{Mn}$ doping in the sample synthesized at $160^{\circ} \mathrm{C}$. Also shown is the calculated XAS for $\left(\mathrm{MnS}_{4}\right)^{6-}$ tetrahedral cluster as described in the text. doped sample synthesized at $60^{\circ} \mathrm{C}$ shows a broad peak centred at about $500 \mathrm{~nm}$, similar to the one reported earlier (Nag et al 2007). This emission is the result of radiative recombination of the electrons and holes via the surface/defect states present in the nanocrystals. The electrons and holes, after excitation across the band edge, trickle down non-radiatively to the surface states lying in the bandgap region. Radiative de-excitation across the surface states in CdS nanocrystals gives rise to bright yellow-green fluorescence observed at around $500 \mathrm{~nm}$. The fluorescence emission maxima shift towards higher wavelength upon increasing the nanocrystal size due to the fact that the bandgap decreases upon increasing the size consequently leading to a decrease in the energy difference between the surface states through which the electrons and holes de-excite radiatively.

Mn $d$ levels provide a channel for de-excitation of electrons and holes other than the surface states. As the Mn $d$ levels lie in the bandgap region they can readily capture the excited electrons from the the band edge as well as surface states (Sapra et al 2005). The characteristic $\mathrm{Mn}^{2+}$ emission occurs at $\sim 590 \mathrm{~nm}$ owing to the ${ }^{4} T_{1}-{ }^{6} A_{1}$ transition. Doping $\mathrm{CdS}$ nanocrystals with $\mathrm{Mn}^{2+}$ ions, synthesized at $60^{\circ} \mathrm{C}$ gives rise to a second peak in the emission spectrum as seen in the upper panel of figure 6 , similar to the one reported earlier (Nag et al 2007). Thus, we know

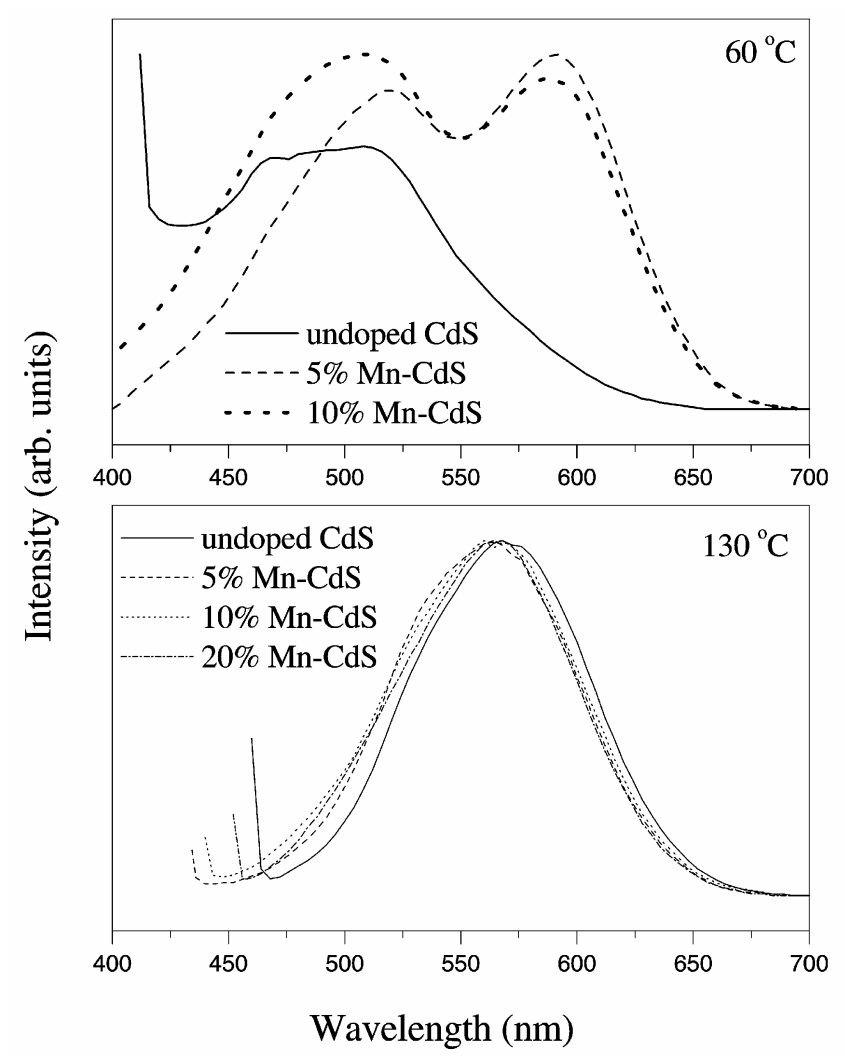

Figure 6. Fluorescence spectra for undoped and Mn-doped $\mathrm{CdS}$ nanocrystals synthesized at $60^{\circ} \mathrm{C}$ and $130^{\circ} \mathrm{C}$. 
that $\mathrm{Mn}^{2+}$ ions are doped into the CdS lattice as the $\mathrm{Mn} d$ levels couple to the CdS electronic states and the excited electrons de-excite via the $\mathrm{Mn} d$ states giving rise to a bright orange fluorescence. As the size of the $\mathrm{Cd}_{1-x} \mathrm{Mn}_{x} \mathrm{~S}$ nanocrystals increases upon increasing the temperature, the fluorescence emission through the surface states shifts to higher wavelengths. This leads to an overlap of the Mn $d$ levels with the surface states and thus we do not observe any separate, well-resolved Mn emission peaks in samples synthesized at $130^{\circ} \mathrm{C}$. The results are different from those reported earlier. Levy et al (1996) observe the surface state emission at wavelengths higher than $590 \mathrm{~nm}$. This cannot be attributed to quantum confinement, since their nanocrystals are of the same size as reported here. We notice a difference in the synthesis methods. Levy et al (1996) used an excess of sulfide ions whereas we used an excess of cadmium ions. Thus, it is reasonable to argue that dangling cadmium bonds, as are present in our case are shallow surface states and thus exhibit emission at a higher energy whereas dangling sulfur bonds are rather deep states that give rise to red-shifted emission. Counio et al (1998) used stoichiometric amounts of cadmium and sulfur ions. They also observed an emission band at an energy lower than that of Mn emission. They attribute it to deep surface trap recombinations which are most likely from defect related states. Thus, in the present system, it is quite likely that the Mn steals some emission intensity from the surface states at higher energy as has been reported for the case of $\mathrm{Zn}_{1-x} \mathrm{Mn}_{x} \mathrm{~S}$ nanocrystals recently (Sapra et al 2005).

To study the nature of the various states involved in fluorescence, lifetime decay studies are very useful. However, it is difficult to isolate the contribution from the various decay channels present in $\mathrm{Cd}_{1-x} \mathrm{Mn}_{x} \mathrm{~S}$ nanocrystals, since both the surface states and the $\mathrm{Mn} d$ levels emit at the same energy for most of the samples reported in the literature so far. In the case of $\mathrm{Zn}_{1-x} \mathrm{Mn}_{x} \mathrm{~S}$ nanocrystals (Sapra et al 2005), the emissions from surface states and the Mn $d$ levels occur at very different energies. For $\mathrm{Cd}_{1-x} \mathrm{Mn}_{x} \mathrm{~S}$ nanocrystals, we have been able to separate the two emissions by synthesizing smaller sized particles by carrying out the reaction at $60^{\circ} \mathrm{C}$. Thus, the surface state emission, which is around $570 \mathrm{~nm}$ for the samples synthesized at higher temperatures, moves to $510 \mathrm{~nm}$ in the case of samples synthesized at $60^{\circ} \mathrm{C}$ as seen in figure 6 . We have carried out fluorescence decay studies for the samples synthesized at $60^{\circ} \mathrm{C}$ with varying $\mathrm{Mn}$ content. We discuss the results of these measurements. Figure 7 shows the fluorescence decay curves of the undoped, 5 and $10 \%$ doped $\mathrm{Cd}_{1-x} \mathrm{Mn}_{x} \mathrm{~S}$ nanocrystals measured at an emission wavelength of $510 \mathrm{~nm}$. This corresponds to the peak of the surface state emission in the samples synthesized at $60^{\circ} \mathrm{C}$. Here we notice the shortening of the $510 \mathrm{~nm}$ fluorescence emission lifetime upon doping the $\mathrm{CdS}$ samples with $\mathrm{Mn}^{2+}$ ions. The shortening of lifetime is less compared to the case of $\mathrm{Zn}_{1-x} \mathrm{Mn}_{x} \mathrm{~S}$ (Sapra et al
2005). This is possibly due to a larger overlap of the emission spectra of the Mn $d$ levels and the surface states in the case of $\mathrm{Cd}_{1-x} \mathrm{Mn}_{x} \mathrm{~S}$. Since the energy of the two emission channels is not so different, the extra stabilization gained by transferring an excitation to $\mathrm{Mn} d$ levels is not large here compared to the case of $\mathrm{Zn}_{1-x} \mathrm{Mn}_{x} \mathrm{~S}$, where the two energies were widely separated. However, some quenching of the surface state emission by $\mathrm{Mn}$ is still observed

Figure 8 shows the fluorescence decay at $590 \mathrm{~nm}$ corresponding to the peak of the $\mathrm{Mn} d$ fluorescence emission. Apart from minor variations, the fast component of the fluorescence decay at $590 \mathrm{~nm}$ is the same in both the doped and undoped samples. Thus, this fast component is only due to the surface states in case of $\mathrm{Cd}_{1-x} \mathrm{Mn}_{x} \mathrm{~S}$ nanocrystals similar to the earlier report (Sapra et al 2005) of $\mathrm{Zn}_{1-x} \mathrm{Mn}_{x} \mathrm{~S}$ nanocrystals and $\mathrm{Mn} d$ levels have no contribution towards this fast component. It is interesting to note that at $590 \mathrm{~nm}$, the shortening of lifetime upon doping is much less compared to that in figure 7. Obviously the extent of overlap between surface state emissions and $\mathrm{Mn} d$ levels is much larger at $590 \mathrm{~nm}$ than that at $510 \mathrm{~nm}$; this further supports the previously made assumption that energy transfer between states reduces upon increase of overlapping of the involved states because of the lack of extra stabilization, normally achieved by the process. However, the lifetime data is not conclusive and to establish the slow decay from Mn $d$ levels we have recorded a time delay spectrum. The intensity of the time delay spectrum is integrated between 100 nanoseconds and 1 microsecond after the excitation pulse. Thus, the fast processes that would have taken place within the first 100

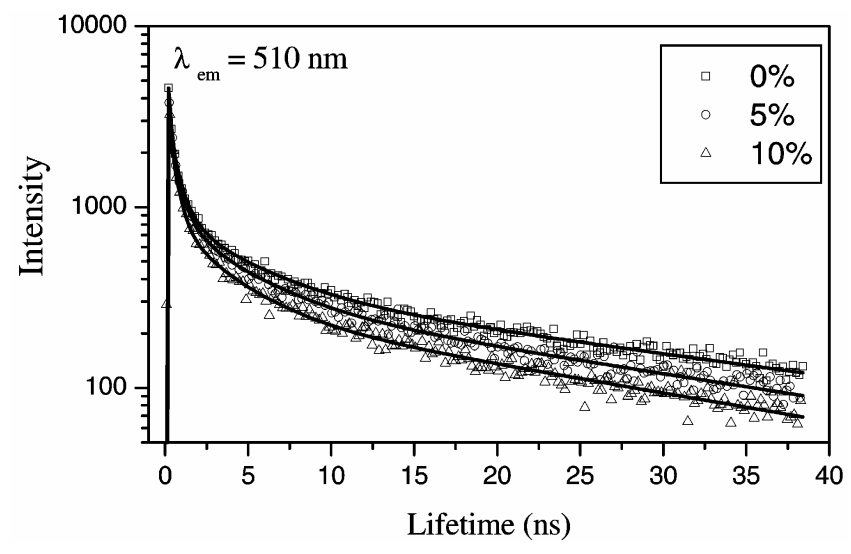

Figure 7. Fluorescence lifetime decay spectra recorded at $510 \mathrm{~nm}$ for undoped, $5 \%$ and $10 \% \mathrm{Mn}$-doped CdS nanocrystals synthesized at $60^{\circ} \mathrm{C}$. The data were recorded at a resolution of $0.039 \mathrm{~ns} / \mathrm{channel}$. The PL decays were best fitted $\left(\chi^{2}\right.$ values $<1.2$ ) to four exponential functions. The five lifetimes (and amplitudes) are as follows: undoped CdS, 0.10 (0.65), 0.61 $(0.24), 4.18(0.07), 34.88(0.04) ; 5 \%$ Mn-doped CdS, 0.09 (0.68), $0.59(0.22), 3.76(0.07), 30.08(0.04) ; 10 \%$ Mn-doped $\mathrm{CdS}, 0.06(0.72), 0.50(0.21), 3.45(0.05), 28.02(0.03)$. 


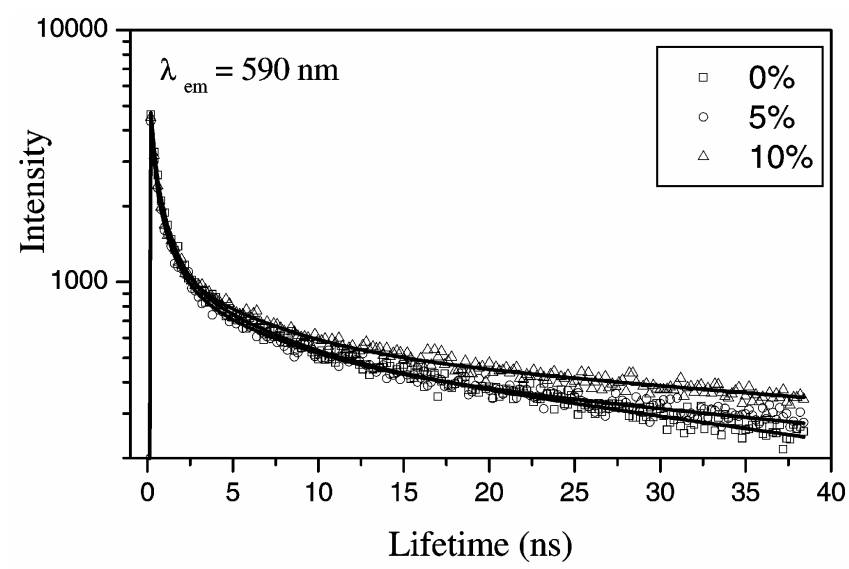

Figure 8. Fluorescence lifetime decay spectra recorded at $590 \mathrm{~nm}$ for undoped, $5 \%$ and $10 \% \mathrm{Mn}$-doped CdS nanocrystals synthesized at $60^{\circ} \mathrm{C}$. The data were recorded at a resolution of $0.039 \mathrm{~ns} /$ channel. The PL decays were best fitted $\left(\chi^{2}\right.$ values $<1.2$ ) to four exponential functions. The five lifetimes (and amplitudes) are as follows: undoped CdS, 0.14 (0.53), 0.78 $(0.30), 4.67(0.90), 45.30(0.08) ; 5 \%$ Mn-doped CdS, 0.16 $(0.58), 0.85(0.26), 5.45(0.09), 66.70(0.07) ; 10 \%$ Mn-doped CdS, $0 \cdot 11(0 \cdot 57), 0 \cdot 72(0 \cdot 28), 5 \cdot 19(0 \cdot 08), 81 \cdot 64(0 \cdot 07)$

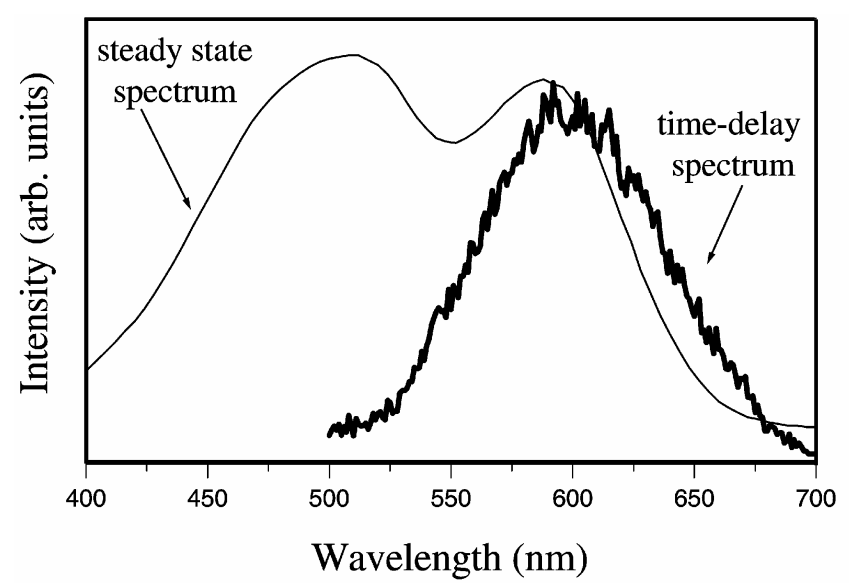

Figure 9. Steady state and time-delay fluorescence spectra for $5 \% \mathrm{Mn}$-doped $\mathrm{CdS}$ nanocrystals synthesized at $60^{\circ} \mathrm{C}$.

nanoseconds are not observed in this spectrum. A comparison of the steady state spectrum with a spectrum recorded after a time delay is shown in figure 9 for the $5 \%$ Mn-doped CdS sample synthesized at $60^{\circ} \mathrm{C}$. The time delay spectrum agrees quite well with the $\mathrm{Mn} d-d$ transition that causes emission at $590 \mathrm{~nm}$ with a complete absence of the $510 \mathrm{~nm}$ emission feature. Thus, the slow component of emission is attributed to $\mathrm{Mn} d-d$ transition. The fast component is the one that is seen in the steady state spectrum, but is absent in the time-delay spectrum. Thus, the fast component corresponds to the surface state emission centred at $510 \mathrm{~nm}$. This clearly shows that Mn has a slow decay rate as in the bulk (Ehrlich et al 1985) and that doping in nanocrystals does not shorten its decay time.

\section{Conclusions}

We have synthesized different sized $\mathrm{Cd}_{1-x} \mathrm{Mn}_{x} \mathrm{~S}$, ranging from $18-33 \AA$ by merely varying the synthesis temperature with varying $\mathrm{Mn}$ concentrations. Although, the starting $\mathrm{Mn}$ concentration is kept very high, a very small fraction of $\mathrm{Mn}^{2+}$ ions are incorporated into the CdS lattice. The EPR and XAS results show that $\mathrm{Mn}^{2+}$ ions are present in the nanocrystals, both inside the lattice and on the surface. With increasing temperatures, the $\mathrm{Mn}^{2+}$ ions shift towards the surface of the nanocrystals. The nanocrystals exhibit fast surface state and Mn $d$ levels related long-lived fluorescence. Fluorescence decay profiles show that the lifetime of the surface state emission reduces upon doping, however, extent of shortening of the lifetime decreases with increased overlapping of the surface state emission with $\mathrm{Mn} d$ levels.

\section{Acknowledgements}

The authors thank the Department of Science and Technology for funding the project. One of the authors (DDS) acknowledges the National J. C. Bose Fellowship. (AN) acknowledges CSIR, Government of India, for a fellowship.

\section{References}

Bhargava R N, Gallagher D, Hong X and Nurmikko A 1994 Phys. Rev. Lett. 72416

Bocquet A E, Mizokawa T, Saitoh T, Namatame H and Fujimori A 1992 Phys. Rev. B 463771

Counio G, Gacoin T and Boilot J P 1998 J. Phys. Chem. B 102 5257

Ehrlich C, Busse W, Gumlich HE and Tschierse D $1985 \mathrm{~J}$. Crystal Growth 72371

Erwin S C, Zu L, Haftel M I, Efros A L, Kennedy T A and Norris D J 2005 Nature 43691

Feltin N, Levy L, Ingert D and Pileni M P 1999 J. Phys. Chem. $B \mathbf{1 0 3} 4$

Furdyna J K 1988 J. Appl. Phys. 64 R29

Guinier A 1963 X-ray diffraction (San Francisco, CA: Freeman)

Hoffman D M et al 2000 Solid State Commun. 114547

Levy L, Hochepied J F and Pileni M P 1996 J. Phys. Chem. 100 18322

Mahadevan P and Sarma D D 2000 Phys. Rev. B 617402

Mikulec F V, Kuno M, Bennati M, Hall D A, Griffin R G and Bawendi M G 2000 J. Am. Chem. Soc. 1222532

Nag A, Sapra S, Nagamani C, Sharma A, Pradhan N, Bhat S V and Sarma D D 2007 Chem. Mater. 193252

Ohno H 1998 Science 281951

Ohno H 1999 J. Magn. Magn. Mater. 200110

Pradhan N and Peng X 2007 J. Am. Chem. Soc. 1293339 
Sapra S and Sarma D D 2004 Phys. Rev. B 69165304

Sapra S, Shanthi N and Sarma D D 2002 Phys. Rev. B 66 205202

Sapra S, Nanda J, Anand A, Bhat S V and Sarma D D 2003 J. Nanosci. Nanotechnol. 3392

Sapra S, Prakash A, Ghanghrekar A, Periasamy N and Sarma D D 2005 J. Phys. Chem. B 1091663

Soo Y L, Ming Z H, Huang S W, Kao Y H, Bhargava R N and Gallagher D 1994 Phys. Rev. B 507602 van der Laan G and Kirkman I W 1992 J. Phys.: Condens. Matter 44189

Viswanatha R, Sapra S, SenGupta S, Satpadi B, Satyam P V, Deb B N and Sarma D D 2004 J. Phys. Chem. B 1086303

Vossmeyer T, Katsikas L, Giersig M, Popovic I G, Diesner K, Chemseddine A, Eychmuller A and Weller H 1994 J. Phys. Chem. 987665

Wang Y, Herron N, Moller K and Bein T 1991 Solid State Commun. 7733 